\title{
TCR-like antibodies in cancer immunotherapy
}

\author{
Qinghua He ${ }^{1}$, Zhaoyu Liu', Zhihua Liu', Yuxiong Lai ${ }^{1}$ Xinke Zhou ${ }^{1}$ and Jinsheng Weng ${ }^{2 *}$
}

\begin{abstract}
Cancer immunotherapy has been regarded as the most significant scientific breakthrough of 2013, and antibody therapy is at the core of this breakthrough. Despite significant success achieved in recent years, it is still difficult to target intracellular antigens of tumor cells with traditional antibodies, and novel therapeutic strategies are needed. T cell receptor (TCR)-like antibodies comprise a novel family of antibodies that can recognize peptide/MHC complexes on tumor cell surfaces. TCR-like antibodies can execute specific and significant anti-tumor immunity through several distinct molecular mechanisms, and the success of this type of antibody therapy in melanoma, leukemia, and breast, colon, and prostate tumor models has excited researchers in the immunotherapy field. Here, we summarize the generation strategy, function, and molecular mechanisms of TCR-like antibodies described in publications, focusing on the most significant discoveries.
\end{abstract}

Keywords: T cell receptor, TCR-like antibody, Antibody, Tumor antigen, Immunotherapy

\section{Background}

Cancer immunotherapy has been cited as the greatest scientific breakthrough of 2013 [1]. The core element of this success is antibody therapy. In the last 40 years, more than 74 different antibody-based molecules have been approved for use in clinical treatment in the European Union, the USA, and Japan $[2,3]$. Currently, there are more than 864 antibodies in phase I, II, or III clinical trials, covering a wide spectrum of diseases in the human body [3]. These have demonstrated the powerful and specific effects of antibody therapy in the field of human diseases and prompt us to seek further breakthroughs in this field.

Activated memory plasma cells secrete antibodies that consist of an fragment antigen-binding (Fab) and a fragment crystallizable region $(\mathrm{Fc})$. After binding to the antigen through their highly variable Fab regions, the antibodies can mediate anti-tumor effects through many different mechanisms. Herceptin, the anti-human epidermal growth factor receptor 2 (HER2) antibody, can bind directly to breast cancer cells and inhibit their metastasis through the induction of apoptosis [4]; rituximab, the

\footnotetext{
* Correspondence: jweng@mdanderson.org

Department of Lymphoma and Myeloma, Division of Cancer Medicine, The University of Texas MD Anderson Cancer Center, 1414 Holcombe Boulevard, Houston, TX 77030, USA

Full list of author information is available at the end of the article
}

anti-cluster of differentiation 20 (CD20) chimeric antibody, can induce lymphoma cell death through antibodydependent cellular cytotoxicity (ADCC) or complementdependent cytotoxicity (CDC) [5]; Opdivo (nivolumab, anti-PD-1), Keytruda (pembrolizumab, anti-PD-1), Yervoy (ipilimumab, anti-CTLA-4), and Kymriah (tisagenlecleucel, anti-CD19 CAR-T cells) can induce tumor lysis through immune cell activation and recruitment [3]; and Mylotarg (gemtuzumab ozogamicin, anti-CD33 antibody-drug conjugate), Adcetris (brentuximab vedotin, anti-CD30 antibody-drug conjugate), and Kadcyla (ado-trastuzumab emtansine, anti-HER2 antibody-drug conjugate) can induce tumor death through conjugated cytotoxin delivery [6]. All of these have attested to the dramatic effects of antibody therapy against cancer cells. However, one limitation of traditional antibody therapy is that the antibodies can target only cell surface antigens and have no effect on intracellular proteins.

Most tumor-specific antigens that control cell growth, proliferation, and death are intracellular. To target these antigens, a specific group of antibodies called $\mathrm{T}$ cell receptor (TCR)-like/mimic antibodies has been developed for clinical therapy [7]. The intracellular tumor-specific antigens can go through the major histocompatibility complex (MHC) class I signaling pathway and present as tumor-specific peptide/MHC complexes on the tumor 


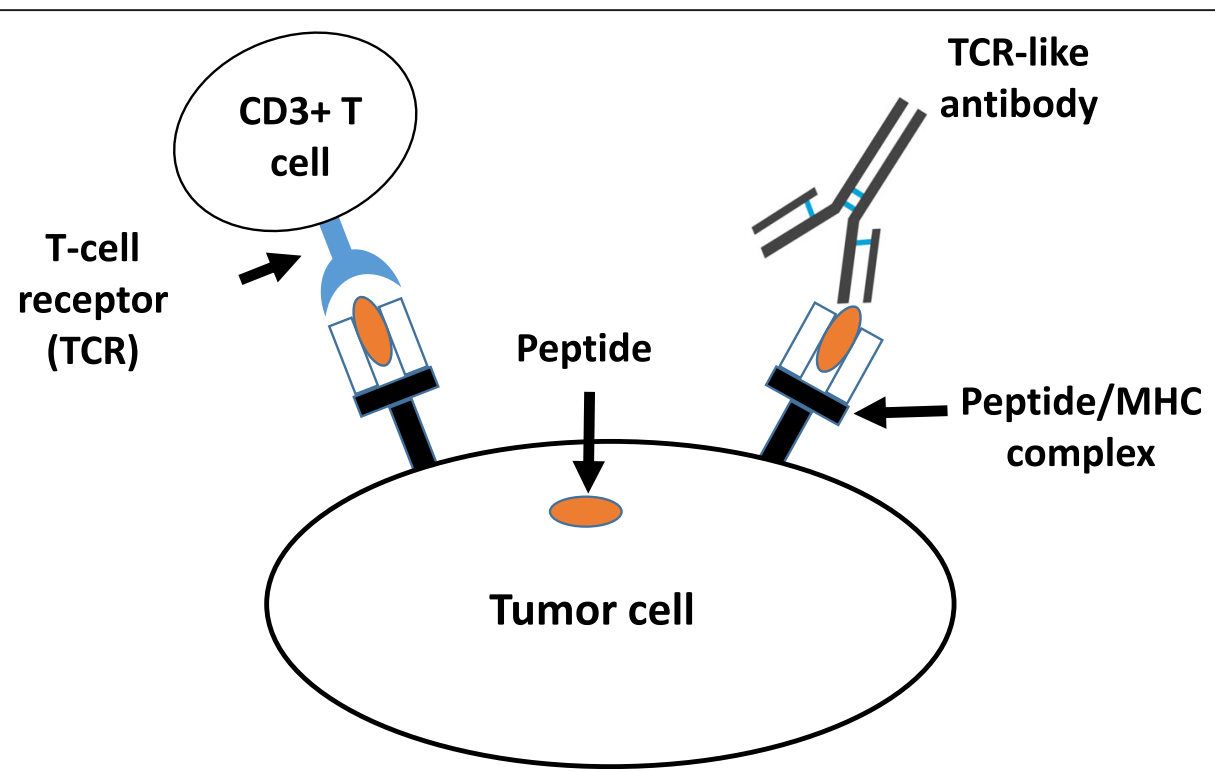

Fig. 1 Schematics of T cell receptor (TCR) and TCR-like receptor. Both TCR and TCR-like antibodies recognize the peptide/MHC complex on the surface of tumor cells

cell surfaces [8]. TCR-like antibodies recognize the peptide/MHC complexes on the tumor cell surfaces in the same manner as authentic TCRs (Fig. 1). The recognition of the peptide/MHC complex by TCRs expressed on the surface of $\mathrm{T}$ cells can trigger various effects, such as $\mathrm{T}$ cell proliferation and differentiation and cytokine or chemokine secretion [9]. The recognition of the peptide/MHC complex by TCR-like antibodies, however, can trigger much broader pharmacological pathways than that of the TCRs in T cells [7]. TCR-like antibodies can trigger ADCC, CDC, antibody-dependent cellular phagocytosis (ADCP), or the direct induction of apoptosis [10]. In addition, TCR-like antibodies can be converted to a chimeric antigen receptor (CAR) structure to mediate the specific recognition of tumor cells by $\mathrm{T}$ cells, such as CAR-T cells [11].

Soluble TCRs have proven to be difficult to engineer in vitro, and their inherently low affinity for their targets limits their use as a single molecular tool to detect the expression of the peptide/MHC complex on the tumor cell surface [12-14]. To overcome these limitations, TCR-like antibodies have been developed as an in vitro tool. For example, TCR-like antibodies have been conjugated with fluorescent reagents to detect the expression level of the Wilms tumor 1 (WT1) RMFPNAPYL peptide/human leukocyte antigen (HLA)-A2 complex on the leukemia cell surface, which offers a clear map of the tumor-specific antigen profile [15]. TCR-like antibodies can also be conjugated with cytotoxic organic compounds, such as antibody-drug conjugates (ADCs), radionuclides, and protein toxins, to mediate the specific killing of tumor cells [16]. Furthermore, immunomodulators or secondary antibodies can be conjugated with the TCR-like antibodies to mediate specific immune responses around the tumor site, as in bi-specific $\mathrm{T}$ cell engagers (BiTE) [17]. Finally, in comparison with the tedious process of preparing a large number of tumor antigen-specific T cells for each patient, as in CAR-T or TCR-T therapy, TCR-like antibodies can be prepared in large amounts, stored for long periods of time, and used as off-the-shelf products for patients, significantly reducing clinical costs. Hence, the research on TCR-like antibodies in the field of tumor therapy has exploded over the last decades $[10,18,19]$.

There are currently more than 40 TCR-like antibodies in pre-clinical development, and most of them show strong anti-tumor effects both in vitro and in vivo (Table 1). Here, we summarize the most significant discoveries for TCR-like antibodies, including the antigen selection, generation strategy, function, and molecular mechanisms of TCR-like antibodies, the advantages and disadvantages of TCR-like antibodies versus other immunotherapies, and future directions for TCR-like antibody development.

\section{Tumor antigen selection}

Tumor antigens are grouped into several categories according to their origins and specificity. The first category is oncovirus antigens, which include Epstein-Barr nuclear antigen 1-3 (EBNA 1-3), latent membrane protein 1 (LMP1), and LMP2 derived from Epstein-Barr virus (EBV) [54], hepatitis $B$ virus $X$ protein (HBX) from hepatitis $B$ virus $(\mathrm{HBV})[55,56]$, core nonstructural protein 3 (NS3) and nonstructural protein 5A (NS5A) from hepatitis $\mathrm{C}$ virus (HCV) [57], type E5, E6, and E7 


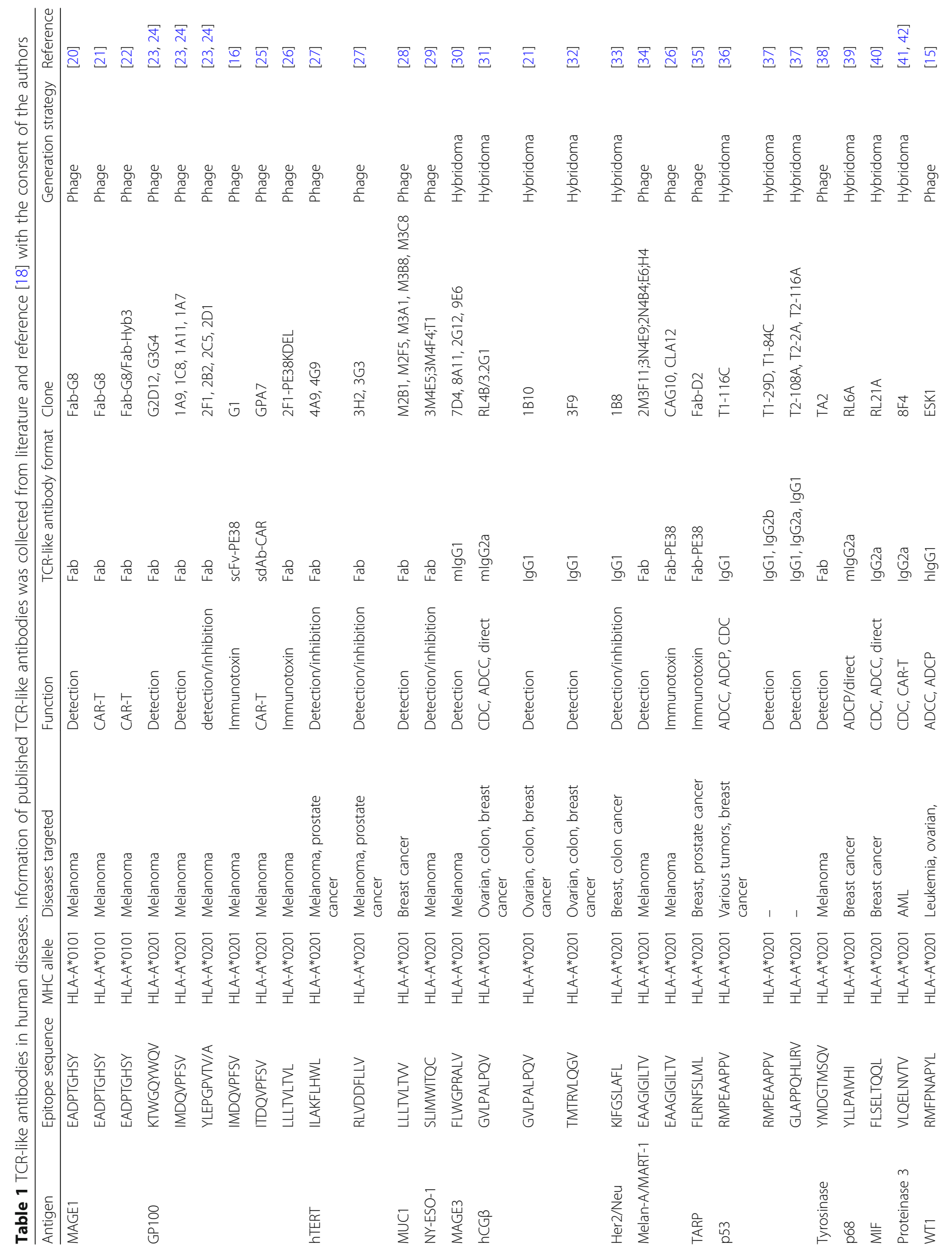




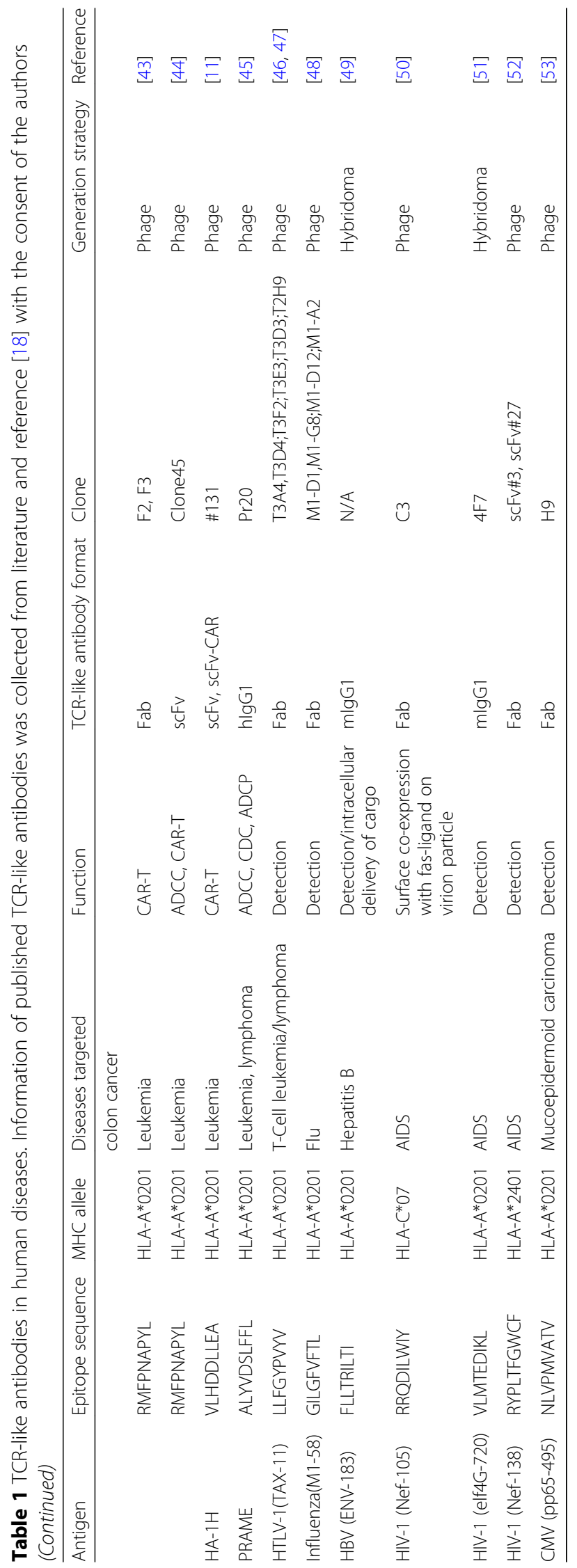


proteins from human papillomavirus (HPV) [58], viral transactivator (Tax) from human $\mathrm{T}$ cell leukemia-lymphoma virus (HTLV) [59], latency-associated nuclear antigen (LANA), virus active $\mathrm{G}$ protein-coupled receptor homolog (vGPCR), and virus IFN-inducible factor (vIRF1) from Kaposi sarcoma-associated herpesvirus (KSHV) [60], structural protein PP65 from cytomegalovirus (CMV) [61], and group-specific antigen (gag) and pol reading frame 468 (Pol468) from human immunodeficiency virus (HIV) [62]. The oncoviruses can cause many diseases, including Burkitt's lymphoma (BL), non-Hodgkin's B cell lymphoma (NHL), nasopharyngeal carcinoma (NPC), hepatocellular carcinoma (HCC), cervical cancer, adult $\mathrm{T}$ cell leukemia (ATL), primary effusion lymphoma (PEL), Kaposi's sarcoma (KS), and Merkel cell carcinoma (MCC). The oncovirus antigens are highly tumor-specific, as they are unique to the oncoviruses and are not shared by normal human tissues. However, viral infections cause only about $10-15 \%$ of all human cancers, and some healthy individuals do not develop cancer even with the infection of an oncovirus [60, 63, 64]. Hence, the oncovirus antigens are of limited use in the clinic.

The second group of tumor antigens involves chromosome/gene mutations in cancer cells $[65,66]$. These mutations include chromosomal translocation, loss, duplication, and loss or point mutation of nucleic acids in the exons, introns, or regulatory regions of genes [67]. These mutations can lead to the expression of truncated proteins, fusion proteins, or neoantigens that are unique to cancer cells, such as beta-catenin S37F in melanoma [68], alpha-actinin-4 K122N in lung cancer [69], heat shock protein 70 kilodalton-2 (hsp70-2) F293I in renal cancer [70], Kirsten rat sarcoma viral oncogene (K-ras) G12D in colon cancer [71], myeloid differentiation primary response 88 (MYD88) L265P in hairy cell leukemia [72], and B cell receptor-Abelson murine leukemia viral oncogene homolog 1 (BCR-ABL) fusion protein in chronic myeloid leukemia [73]. These antigens are tumor cell-specific. However, some types of cancer have a high burden of genetic mutations, whereas other types of cancers may not; in addition, many genetic mutations are unique to the tumor cells of individual patients [74, 75]. Hence, this group of tumor antigens is difficult to target with the current adoptive cellular therapy strategy.

The third group of tumor antigens is the cancer-testis antigens, which are overexpressed in multiple types of tumor cells of patients $[76,77]$. In healthy donors, this group of antigens is expressed only in immune-privileged organs, such as the testis or placenta. Because the immune-privileged organ cells do not express $\mathrm{MHC}$ alleles, TCRs that recognize the peptide/MHC complex derived from this group of antigens will not damage the normal tissue cells [78]. Moreover, high-affinity TCRs targeting cancer-testis antigens can be isolated from the peripheral blood of normal donors because of the absence of cancer-testis antigens in the peripheral blood $[79,80]$. Hence, this group of tumor antigens, including New York esophageal squamous cell carcinoma-1 (NYESO-1), melanoma-associated antigen A (MAGE-A), and synovial sarcoma $\mathrm{X}$ (SSX), comprises the largest number in current clinical trials [81, 82].

The fourth group of tumor antigens involves antigens with minimal or limited expression in normal cells, such as carcinoembryonic antigen (CEA), melanoma antigen recognized by $\mathrm{T}$ cells 1 (MART-1), and tyrosine kinase 10 [83-85]. Targeting these antigens can damage normal tissues, and sophisticated technology is needed for the future development of immunotherapy against these antigens [86, 87]. This group also includes antigens derived from non-essential organs, such as CD19 and CD20 from $B$ cells [88]. Targeting these antigens can cause non-fatal damage to normal tissue, which medical interventions can cure [89, 90].

Importantly, about $95 \%$ of the aforementioned tumor antigens are intracellular proteins, and very few tumorspecific antigens are extracellular [91]. Thus, to target tumors through tumor-specific antigens, a novel strategy must be developed.

\section{TCR-like antibody generation}

Because intracellular proteins can be digested into small peptides in the proteasome of a cell, which can be conjugated with MHC molecules in the endoplasmic reticulum (ER) and transported to the tumor cell surface, the peptide/MHC complex on the tumor cell surface has been deemed as a tumor-specific antigen [92]. MHC class I molecules are expressed on the surface of all nucleated cells, and numerous studies have demonstrated the feasibility of targeting tumors through the recognition of the peptide/MHC complex on the cell surface $[85,93,94]$.

In 1981, Wylie and Klinman conducted the first study of a TCR-like antibody [95]. To study the immune response to influenza, they injected influenza virus and the virus-infected cell line PR8-L929 into C3H/HeJ and BAL6.K mouse strains. They found that approximately one third of the virus-specific antibodies reacted to viral hemagglutinin (HA) or neuraminidase. The remainder of virus-specific antibodies recognized antigens found on the surface of virus-infected PR8-L929 cells but not on the virion or uninfected cells. It was later found that the MHC participated in the recognition of viral antigens by the antibodies [96]. Similar results have been found in mouse cells transformed with simian virus antigen (SV40), murine cytomegalovirus (MCMV) pp89 (168-176) peptides, vesicular stomatic virus (VSV), and EBV [97-99]. It was demonstrated that mouse MHC conformational epitopes are peptide- 
specific. The monoclonal antibody (mAb) 34.4.20 recognized the VSV nucleoprotein (52-59) peptide on mouse $\mathrm{H}-2 \mathrm{~Kb}$ but not ovalbumin (OVA) (257-264), MCMV pp89 (168-176), or influenza nucleoprotein (Y345-360) peptides on the same MHC allele [98]. Although these studies did not test the cytotoxic effect of TCR-like antibodies, they provided clear evidence that TCR-like antibodies generated in the mouse B cells can specifically bind to the peptide/MHC complex on the cell surface.

In 2000, Chames and colleagues reported the first TCR-like antibody targeting human tumor antigens [20]. Using the phage library technique, they isolated a human antibody directed against the EADPTGHSY peptide encoded by MAGE-A1 and presented by the HLA-A1 molecule. $M A G E-1$ is a cancer-testis gene overexpressed in multiple cancers but with restricted expression in the testis of a healthy person [100]. The phage Fab antibody bound to the HLA-A1 molecule complexed with the MAGE-A1 peptide but not to the HLA-A1 molecule complexed with other peptides, indicating the specificity of the antibody. Furthermore, the TCR-like antibody bound to the MAGE-1+/HLA-A1+ melanoma cells, indicating that the phage library-derived Fabs could recognize the native complex displayed on the surface of tumor cells. Compared to mouse hybridoma technology, the phage library screening is structure-dependent, fast, and cost-effective. This technique was subsequently explored in the study of TCR-like antibodies against peptide/MHC complexes derived from other tumor antigens, such as telomerase catalytic subunit [27], glycoprotein 100 (gp100) [23, 24], mucin 1 (MUC1) [28], human telomerase reverse transcriptase (hTERT) [27], NYESO1 [29], MART-1 [34], preferentially expressed antigen in melanoma (PRAME) [45], tyrosinase [38], and WT1 [15]. TCR-like antibodies targeting virus epitopes derived from HTLV [46, 47], influenza [48], HIV [50, 52], and CMV [53] were also developed through the phage library strategy.

Early studies of these phage library-derived Fab antibodies focused on the use of antibodies as tools to detect the expression levels of peptide/MHC complexes on the tumor cell surfaces. To develop therapeutic strategies with phage library-derived TCR-like antibodies, researchers have used the CAR strategy by ligating the heavy chain variable $(\mathrm{VH})$ and light chain variable $(\mathrm{VL})$ region of the phage library-derived Fab antibody with the intracellular domain of CD3 molecules. The first TCR-like CAR-T strategy was developed in 2001 by ligating the $\mathrm{VH}$ and $\mathrm{VL}$ of the Fab antibody targeting the melanoma cells expressing MAGE-A1 and HLA-A1 [21]. The Fab recognizing the MAGE-A1 EADPTGHSY peptide/MHC complex on the melanoma cell surface was fused to the Fc (epsilon)RI-gamma molecule and retrovirally transduced into normal $\mathrm{T}$ cells. The transduced primary human $\mathrm{T}$ lymphocytes bound to the MAGE-A1 peptide/MHC complexes and responded to native $M A G E-A 1+/ H L A-A 1+$ target cells by specific cytokine production of interferon gamma (IFNY) and tumor necrosis factor alpha (TNF $\alpha$ ). These $\mathrm{T}$ cells could also lyse $M A G E-A 1+/ H L A-A 1+$ target cells but not control MAGE-A1-/HLA-A1+ or MAGE-A1+/HLA-A1tumor cells, indicating that the lysis of tumor cells via TCR-like antibodies was HLA-restricted and antigendependent. In a later study, the phage library-purified antibodies were further mutated through a combination of light (L) chain shuffling, heavy $(\mathrm{H})$ chain-targeted mutagenesis, and in vitro selection of phage display libraries to be higher affinity (Fab-Hyb3) [22]. A functional study of Fab-Hyb3 found that the mutated TCR-like CAR-T mediated better recognition of the antigen on the tumor cell surface, indicating that the affinity of TCR-like antibodies dramatically affected the killing ability of the antibodies. The CAR-T technology has since been employed in several other TCR-like antibody studies, including those of gp100, minor histocompatibility antigen $1 \mathrm{H}$ (HA-1H), and WT1 [11, 25, 43, 44].

In 2006, Wittman and colleagues started to use the TCR-like antibody as a typical antibody therapy to mediate ADCC and CDC effects against tumors [31]. To target an HLA-A2-restricted peptide derived from human chorionic gonadotropin beta (hCG- $\beta$ ), which is overexpressed in over $90 \%$ of breast cancers, they developed a mouse IgG2a mAb (termed 3.2G1) via the hybridoma technique. The $3.2 \mathrm{G} 1$ antibody recognized the GVLPALPQV peptide from hCG- $\beta$ presented by the HLA-A2 molecule and specifically stained the cells in a peptide- and antibody concentration-dependent manner. Staining of human tumor lines with the 3.2G1 TCR-like antibody also demonstrated the antibody's ability to recognize endogenously processed peptides from the breast cancer cell line MDA-MB-231. Moreover, 3.2G1 antibody mediated $\mathrm{CDC}$ and $\mathrm{ADCC}$ against the human breast carcinoma MDA-MB-231 cell line in vitro and inhibited tumor implantation and growth in nude mice. These results provided valid evidence for the development of novel therapeutic antibodies that specifically kill tumors via recognition of peptide/MHC complexes. Since then, several TCR-like antibodies have been developed via the hybridoma strategy to mediate ADCC, CDC, or ADCP effects against tumor cells. These include TCR-like antibodies targeting peptide/MHC complexes derived from tumor protein 53 (TP53) [36], macrophage migration inhibitory factor (MIF) [40], proteinase 3 (PR1) [41], and WT1 [15, 44]. In addition to ADCC and CDC effects, the mouse hybridoma-derived TCR-like antibodies can also be utilized therapeutically to detect the expression of peptide/MHC complexes on 
the tumor cell surface as phage library-derived Fab antibodies [30, 32, 33, 37, 49, 51].

Because antibodies can be conjugated with toxins to deliver specific cytotoxic effects into cells, Denkberg and colleagues generated a conjugation molecule with a TCR-like antibody in 2003 [16]. In their study, a singlechain HLA-A2 molecule complexed with a common antigenic $\mathrm{T}$ cell HLA-A2-restricted epitope derived from the gp100 was used to immunize HLA-A2 transgenic mice. A phage display library was constructed from the immunized mice, and a recombinant single-chain fragment variable $(\mathrm{scFv})$ antibody that could bind to the gp100 IMDQVPFSV peptide/MHC complex with a high affinity in the nanomolar range was isolated. When fused to a very potent cytotoxic effector molecule in the form of a truncated bacterial toxin, the TCR-like antibody could specifically kill antigen-presenting cells (APCs) in a peptide-dependent manner. In 2008, Epel and colleagues employed the same technology to fuse a truncated form of Pseudomonas exotoxin A with the phagederived TCR-like antibody that specifically targets the FLRNFSLML peptide/HLA-A2 complex derived from TCR gamma alternate reading frame protein (TARP) [35]. The fusion molecule exhibited specific cytotoxic activity on breast and prostate cancer cells that correlated with their TARP and HLA expression patterns and inhibited the growth of human breast tumor cells in nude mice. These results demonstrated the power of the TCR-like antibody conjugation approach to generate novel targeting molecules to eliminate tumor cells with the unique specificity observed in cytotoxic CD8+ T cells [101]. In the same year, a TCR-like antibody targeting MART-1 conjugated with immunotoxin was also developed for anti-melanoma therapy [26].

TCR-like antibodies can also induce tumor cell death directly after binding to the peptide/MHC complex on the tumor cell surface [102]. In 2006, Verma and colleagues generated two TCR-like antibodies (RL4B and RL6A) that recognized peptides derived from hCG- $\beta$ and human p68 RNA helicase. They found that two TCR-like antibodies destroyed tumor cells independently of immune effector mechanisms, such as ADCC and CDC. TCR-like antibodies mediated the apoptosis of tumor cells through selective and specific binding to $\mathrm{p} 68$ RNA helicase YLLPAIVHI and hCG- $\beta$ GVLPALPQV peptide/HLA class I complexes, which triggered the activation of c-Jun $\mathrm{N}$-terminal kinases (JNKs) and intrinsic caspase pathways. This signaling was accompanied by the release of mitochondrial cytochrome $\mathrm{c}$ and apoptosis-inducing factor. The apoptosis induced by the TCR-like antibodies was completely inhibited by soluble MHC tetramers loaded with relevant peptides and by inhibitors for JNKs and caspases. Thus, their study suggested the existence of a novel mechanism of TCR-like antibodies in the mediation of tumor cell destruction, in addition to ADCC and CDC. This mechanism would appear to be especially important due to the absence or tolerance of immune cells in cancer patients [103-105].

The major functions of TCR-like antibodies include the detection of peptide/MHC complexes, CAR-T strategy, ADCC, CDC, ADCP, immunotoxin targeting, and direct induction of tumor cell death. TCR-like antibodies can also be used as a block to prevent the recognition of normal tissue cells by self-reacting $\mathrm{T}$ cells in autoimmune diseases. In an experimental allergic encephalomyelitis mouse model, Aharoni and colleagues developed several monoclonal antibodies that bound to the complex of myelin basic protein (BP) peptide on mouse I-As [106]. The antibodies blocked the proliferative response of in vitro cultured $\mathrm{T}$ cells to the BP peptide/I-As complex without affecting the $\mathrm{T}$ cell response to an irrelevant peptide derivative from tuberculin on the same allele. The antibodies also inhibited experimental allergic encephalomyelitis in $\mathrm{H}-2 \mathrm{~s}$ mice. Hence, antibodies directed specifically to the autoantigen/MHC complex may offer a highly selective and effective treatment in autoimmune diseases. Moreover, in 2004, Held and colleagues generated a high-affinity $(\mathrm{Kd}=60 \mathrm{nM})$ antibody that specifically recognized the NY-ESO-1 (157-165) but not NY-ESO-1 (157-167) or a cryptic NY-ESO-1 (159-167) peptide/HLA-A2 complex. In a dose-dependent manner, the antibody blocked the recognition of NY-ESO-1/HLA-A2-positive tumor cells by NY-ESO-1 (157-165) peptide-specific CD8+ T cells [29].

\section{Molecular mechanisms of TCR-like antibodies against tumor cells}

Due to the clinical prevalence of cancers, most studies of TCR-like antibodies have been conducted in the field of cancers. The major functions of TCR-like antibodies have been explored in two areas-the detection and measurement of the expression of tumor-specific peptide/MHC complexes on the tumor cell surfaces and the mediation of cytotoxicity against tumor cells. The detailed molecular mechanisms of TCR-like antibodies are summarized as follows (Fig. 2):

- TCR-like antibodies can be conjugated with fluorescent reagents to measure the expression levels of peptide/ MHC complexes on tumor cell surfaces. By using a TCR-like antibody directed against the mucin epitope (HLA-A2-MUC1-D6) with calibration beads, Cohen and colleagues quantified the number of MUC1-D6derived peptide/MHC complexes on the surface of tumor cells to be several hundred per cell [28]. Using TCR-like antibodies against peptide/MHC complexes of three major melanocyte differentiation antigens 


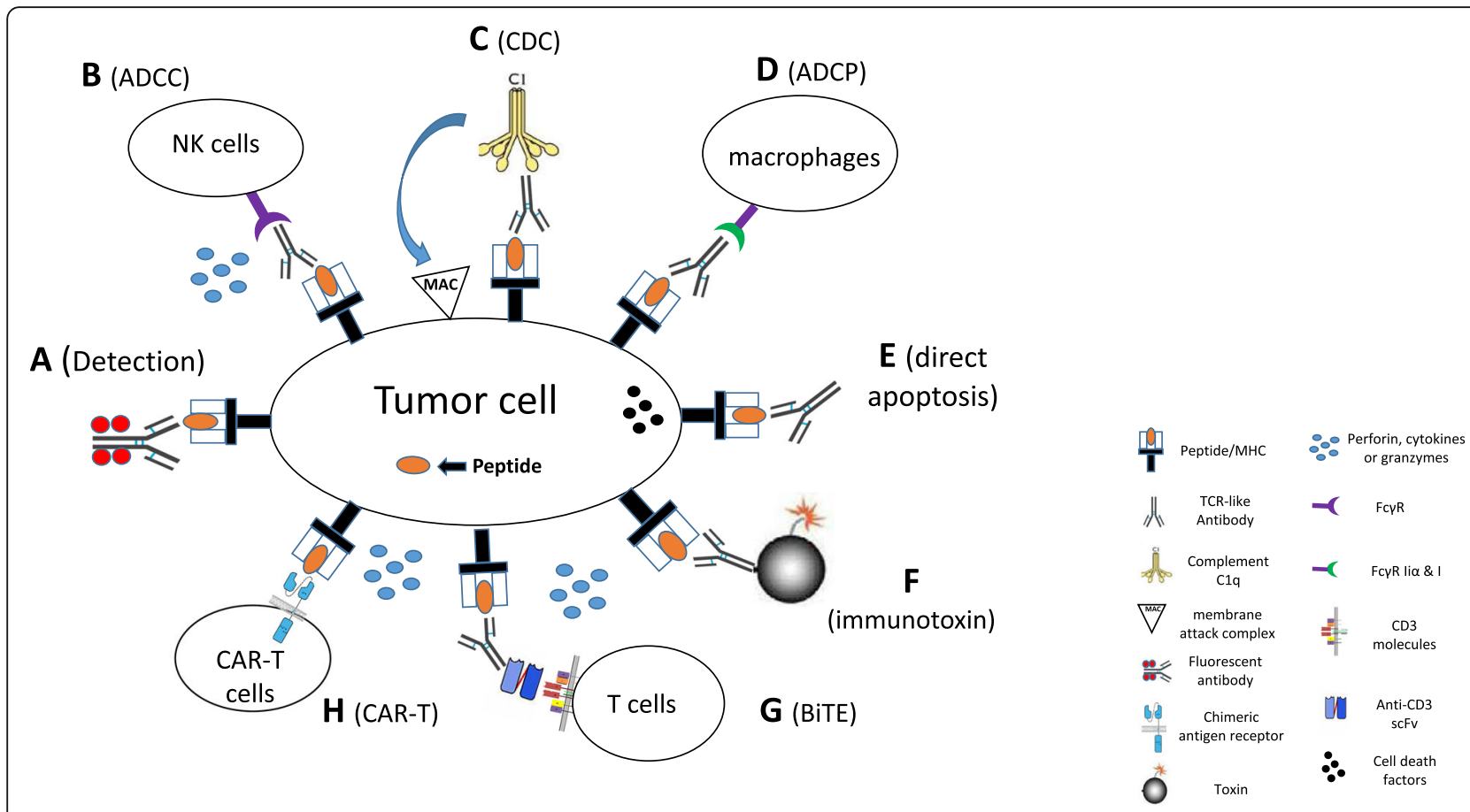

Fig. 2 The molecular mechanisms of TCR-like antibodies against tumor cells. TCR-like antibodies mediate their anti-tumor effects through multiple mechanisms. (a) Conjugation with fluorescent reagents to detect the expression of the peptide/MHC complex on the surface of tumor cells. (b) Antibody-dependent cell-mediated cytotoxicity (ADCC) with NK cells. (c) Complement-dependent cytotoxicity (CDC) with complements. (d) Antibody-dependent cellular phagocytosis (ADCP) with microphages. (e) Direct induction of tumor cell apoptosis. (f) Conjugation with drugs or toxins. (g) Conjugation as a bi-specific T cell engager (BiTE). (h) CAR-T strategy. TCR-like: T cell receptor-like; MHC: major histocompatibility complex; MAC: membrane attack complex; FcyR: Fc gamma receptor; FcyR Ila: Fc gamma receptor II alpha

(gp100, Mart-1, and tyrosinase), Yael and colleagues found that melanoma cell lines had an average of approximately 4000 surface copies per cell of the complexes of HLA-A2 and tyrosinase (369-377) but only a few hundred copies per cell of gp100 and Mart1 complexes [38]. This information about the antigen expression hierarchy is highly valuable when considering immunotherapy targets, because the levels of specific peptide/MHC complexes on tumor cells correlate with their susceptibility to cytotoxic $\mathrm{T}$ lymphocyte (CTL) killing [33].

- TCR-like antibodies can be converted to a CAR structure to mediate specific tumor lysis by $\mathrm{T}$ cells. The VH and VL regions of TCR-like antibodies can be converted to $\mathrm{ScFv}$ and ligated to the intracellular domain of CD3 molecules. After being lentivirally or retrovirally transduced into patients' $\mathrm{T}$ cells, the ScFv region of TCR-like CAR can bind to the peptide/MHC complex on the surfaces of tumor cells. The intracellular domain of the TCR-like CAR can activate multiple cellular signaling pathways that lead to $\mathrm{T}$ cell activation and differentiation and secretion of cytokines, perforin, and granzymes [20, 27]. Many TCR-like antibodies isolated from phage display libraries that are in a monovalent antibody form (Fab or scFv fragments) have been successfully converted to CAR structures, and the TCR-like antibody derived from mouse hybridoma can also be converted [42].

- TCR-like antibodies can be used as a standard antibody therapy against tumor cells through ADCC, CDC, or ADCP. In ADCC, the full-length TCR-like antibodies, after binding to the peptide/ MHC complex with Fab region, can bind to the Fc gamma receptors $(\mathrm{Fc} \gamma \mathrm{R})$ expressed on the surfaces of natural killer (NK) cells, monocytes, macrophages, neutrophils, eosinophils, and dendritic cells. These cells can then be activated to release cytokines and cytotoxic granules or express cell death-inducing molecules [31]. In CDC, the complement component $1 \mathrm{q}(\mathrm{C} 1 \mathrm{q})$ binds to the TCR-like antibody and triggers the complement cascade. This leads to the formation of the membrane attack complex (MAC) (C5b to C9) at the surface of the target cells by the classical pathway of complement activation [41]. In ADCP, the TCR-like antibodies engage the Fc gamma receptors II $\alpha$ (FcyRIIa) and FcyRI expressed on macrophages to trigger a signaling cascade that leads to the engulfment of the tumor cells [36]. 
- TCR-like antibodies can be conjugated with toxins or drugs to specifically lyse tumor cells [16, 35]. After the antibodies bind to cell surface antigens, some tumor cells can engulf the antibodies into the cytoplasm through the process of endocytosis. Diphtheria toxin (DT) or Pseudomonas exotoxin A are the most popular immunotoxins being evaluated in clinical trials because these bacterial toxins are easily produced in E. coli with high activity and few side effects in humans [107]. After being translocated to the cytosol, these toxins can catalyze adenine diphosphate (ADP) ribosylation of a diphthamide residue of elongation factor 2 (EF2), causing arrest of protein synthesis that leads to apoptotic cell death [108].

- TCR-like antibodies can be conjugated with secondary antibodies to specifically activate immune cells. The most commonly used secondary antibody is anti-CD3 scFv antibody, which can activate immune cells in a non-specific way. After binding to the peptide/MHC complex on the tumor cell surfaces, the anti-CD3 scFv-conjugated TCR-like antibodies recruit and activate immune cells to secrete perforin, cytokines, and granzymes at the proximity of tumor cells, leading to lysis of the tumor cells [17].

- TCR-like antibodies can induce tumor cell death directly after binding to the peptide/MHC complex. This effect is mediated by the activation of JNKs and intrinsic caspase pathways, accompanied by the release of mitochondrial cytochrome $\mathrm{c}$ and apoptosis-inducing factor in tumor cells [102]. Interestingly, this effect is not observed with pan anti-HLA antibodies lacking peptide specificity.

It is generally believed that, because of the repetitive antigen stimulation and in vivo selection process of hybridoma technology, TCR-like antibodies isolated using this technology have relatively high binding affinity (low nanomolar range) compared with the moderate to average binding affinity ( 50-300 $\mathrm{nM})$ of phage-derived TCR-like antibodies [31, 41, 53]. However, phage library-derived TCR-like antibodies of high affinity in the low nanomolar range have also been successfully isolated from secondgeneration libraries and by in vitro affinity maturation $[22$, 109]. TCR-like antibodies derived from both technologies have been evaluated in pre-clinical studies.

\section{Advantages and disadvantages of TCR-like antibodies versus other immunotherapies}

The greatest advantage of TCR-like antibodies is their ability to target intracellular tumor antigens with minimal in vitro manipulation. The TCR-T adoptive cell therapy can also target intracellular antigens but requires a much more complicated preparation process [78]. In the traditional TCR-T adoptive cell therapy, the peripheral blood or tumor infiltration lymphocytes from a cancer patient must be isolated by apheresis. The lymphocytes are activated for 1 to 3 days to be transduced by TCR-containing lentivirus, retrovirus, or transposon vectors. The transduced $\mathrm{T}$ cells are then expanded to a large number $\left(1 \times 10^{9}\right)$ before infusion back into the patient. The entire procedure takes about 3 to 4 weeks and is technically demanding, expensive, and time-consuming without the guarantee of success [94]. In addition, the transduced antigen-specific TCRs may mismatch with endogenous wild-type TCRs, as both TCRs exist in the same T cells $[110,111]$. TCR-like antibodies, however, are relatively easy to prepare and store and used as off-the-shelf. Through the binding of the Fab region to the peptide/MHC complex, the Fc region of the TCR-like antibody can bind to the Fc gamma receptors (FcyR) expressed by patients' NK cells, monocytes, or macrophage cells and activate these cells to kill tumors.

CAR- $T$ is a specific form of tumor immunotherapy that equips the $\mathrm{T}$ cells with the tumor surface antigenspecific antibody and CD3 signaling pathway [112]. The recognition of tumor surface antigen by the antibody can trigger the CAR-T cell activation and the killing of tumor cells. The clinical success of CD19 CAR-T cells has proved their dramatic effect against tumors [113115]. There are several reports of converting the TCRlike antibodies, especially the phage library-derived Fab antibodies, into CAR vectors [11, 25, 43]. T cells transduced with TCR-like antibody-derived CARs can specifically lyse tumor cells, indicating the therapeutic effectiveness of TCR-like antibody CAR-T cell therapy. Because of the lack of tumor-specific biomarkers on the surface of tumor cells, the traditional CAR-T therapy has achieved little success in solid tumors [116]. We envision that the TCR-like antibody CAR-T cell therapy could have specific value for solid tumors, as it targets intracellular tumor-specific antigens.

The checkpoint antibody strategy is a significant step in the history of humanity's fight against cancer [117]. The molecular mechanism of this strategy is that the checkpoint antibody can reverse the immune suppression of tumor antigen-specific $\mathrm{T}$ cells that pre-exist in the patient's body so that they may target the cancer cells [103]. The success of CTLA- 4 and PD- 1 checkpoint antibody therapy in the clinic has confirmed this mechanism [118]. However, checkpoint antibody therapy is effective in only about $20-30 \%$ of patients when used individually and $40-60 \%$ of patients when used in combination $[119,120]$. These low rates indicate there may be a lack of tumor antigen-specific $\mathrm{T}$ cells at the tumor site, which hampers the effect of the therapy. TCR-like antibody therapy, however, does not depend on the 
existence of tumor antigen-specific $\mathrm{T}$ cells in the patient's body and can activate the normal immune cells to target the tumor cells through ADCC, CDC, or ADCP $[7,18]$. Combining TCR-like antibodies with checkpoint antibodies in future clinical studies may further improve the responses of patients.

Vaccine therapy is a longtime developed idea in the field of cancer immunotherapy, preceding the CAR-T cellular therapy and checkpoint antibody therapy [121]. The concept of using the host's own immunity to fight cancers in the long-term has attracted significant interest from the scientific community. However, only two vaccines have currently been approved to treat cancer patients, and most tumor vaccines have shown poor clinical results, leading to their failure to secure approval from the US Food and Drug Administration (FDA) [122, 123]. It is hypothesized that the effect of a tumor vaccine is dependent on the development of memory immunity of tumor-specific $\mathrm{T}$ cells, and the tumor environment is usually plagued with immune-suppressive molecules [124]. Thus, it is difficult to induce a strong anti-tumor effect by the vaccine strategy. Moreover, the vaccine strategy is time-consuming and may take several months to develop tumor antigen-specific T cells. TCR-like antibodies, however, do not depend on the existence of tumor antigen-specific $\mathrm{T}$ cells and can take effect immediately after administration.

\section{The future of TCR-like antibody therapy}

TCR-like antibodies, as new tools in the cancer immunotherapy field, have just begun to attract attention from the scientific community. By combining their fine specificity to recognize the peptide/MHC complexes of $\mathrm{T}$ cells with the biological and pharmacological properties of an antibody, TCR-like antibodies may have broad applications in the clinic. However, there are also several hurdles that must be overcome to achieve clinical success with the TCR-like antibodies.

First, TCR-like antibodies are MHC-restricted, which means that they are effective only for a certain group of patients expressing the tumor-specific antigen on a specific MHC allele. With HLA-A2 as the most common $\mathrm{MHC}$ allele in cancer patients, many tumor-specific peptides associated with this allele have been found [91]. Other HLA alleles, however, still lack tumor-specific peptides, which hamper the development of TCR-like antibody therapy. Further identification of less-common MHC-associated peptides will help solve this problem.

Second, the downregulation or absence of peptide/ MHC complexes on the tumor cell surface is a common mechanism of tumor cells to evade immune surveillance [125]. TCR-like antibodies, like TCR-T therapy, may suffer from this effect. However, reports showed that some chemicals, cytokines, or radiation therapy can upregulate the expression of $\mathrm{MHC}$ and activate the $\mathrm{MHC}$ signaling pathway [126, 127]. Thus, TCR-like antibodies may combine with other therapies to achieve the best results. Furthermore, the affinity of TCR-like antibodies is generally higher than the affinity of in vitro synthesized TCRs [43]. The affinity of TCR-like antibodies can also be easily mutated to a higher affinity via molecular technology [22]. This will render antibodies more capable of recognizing the peptide/MHC molecule at extremely low levels.

Third, the immune-suppressive environment is a hurdle for the TCR-like antibody immunotherapy. Tumor cells reside in hidden sites to prevent the access of $\mathrm{T}$ cells, generate a hypoxic environment, and secrete a large amount of immune-suppressive cytokines, such as interleukin 10 (IL-10), transforming growth factor beta (TGF- $\beta$ ), or other molecules that cause the T cells, NK cells, macrophages, or monocyte to experience anergy or death $[124,128]$. In addition, there are many suppressive immune cells around the tumor cells, which dampen the anti-tumor immune response $[129,130]$. Thus, TCR-like antibodies may bind to the peptide/MHC complex on the tumor cell surface but might not mediate tumor destruction. Combining the TCR-like antibody therapy with other immune suppression-reversion therapy could help solve this problem. Examples may include the adoptive transfer of freshly expanded NK cells, monocytes, or macrophages in combination with TCR-like antibody therapy, or the combination of anti-PD-1 or anti-CTLA-4 antibody therapy. One advantage of TCRlike antibodies is that they can easily penetrate the tumor environment and they do not require the existence of tumor antigen-specific $\mathrm{T}$ cells at the tumor site. Furthermore, some of the TCR-like antibodies can induce tumor cell death directly through binding to the peptide/MHC complex [39, 102].

\section{Conclusion}

With only a few dozen TCR-like antibodies reported in publications, we have limited knowledge about this new group of antibodies. However, with solid evidence of their effectiveness in hematological and non-hematological preclinical tumor models and unique character to detect the expression levels of tumor-specific peptide/MHC complexes on the surface of tumor cells, TCR-like antibodies may represent an ideal next step for cancer immunotherapy.

\section{Abbreviations}

ABL: Abelson murine leukemia viral oncogene homolog 1; ADC: Antibodydrug conjugate; ADCC: Antibody-dependent cellular cytotoxicity; Adcetris: Brentuximab vedotin, abti-CD30 antibody-drug conjugate; ADCP: Antibody-dependent cellular phagocytosis; APCs: Antigen-presenting cells; ATL: Adult T cell leukemia; BCR: B cell receptor; BiTEs: Bi-specific T cell engaging antibodies; BL: Burkitt's lymphoma; BP: Basic protein from myelin; C1q: Complement component 1q; CAR: Chimeric antigen receptor; CART: Chimeric antigen receptor T cells; CD19: Cluster of differentiation 19; 
CD20: Cluster of differentiation 20; CD3 द: CD3 zeta; CDC: Complementdependent cytotoxicity; CEA: Carcinoembryonic antigen;

CMV: Cytomegalovirus; CTLA-4: Cytotoxic T-lymphocyte-associated protein-4; DT: Diphtheria toxin; E5: Papillomavirus E5 antigen; E6: Papillomavirus E6 antigen; E7: Papillomavirus E7 antigen; EBNA: Epstein-Barr nuclear antigen; EbV: Epstein-Barr virus; ER: Endoplasmic reticulum; F293I: Phenylalanine mutated to isoleucine at 293 position; Fab: Fragment antigen-binding; FC: Fragment crystallizable region; FcyR Ila: Fc gamma receptor II alpha; FcyR: Fc gamma receptor; FDA: US Food and Drug Administration; G12D: Glycine mutated to aspartic acid at 12 position; $\mathrm{H}$ chain: Immunoglobulin heavy chain; HA: Viral hemagglutinin; HBV: Hepatitis $B$ virus; HBX: Hepatitis B virus X protein; HCC: Hepatocellular carcinoma; HCV: Hepatitis C virus; HER2: Human epidermal growth factor receptor 2; HIV: Human immunodeficiency virus; HIV.Gag: Group-specific antigen from HIV; HIV-Pol468: Pol reading frame 468 from HIV; HLA: Human leukocyte antigen; HPV: Human papillomavirus; hsp70-2: Heat shock protein 70-2; hTERT: Telomerase reverse transcriptase; HTLV: Human T cell leukemialymphoma virus; I-As: Mouse MHC allele; IFN-ץ: Interferon gamma; JNK: C-Jun $\mathrm{N}$-terminal kinases; K122N: Lysine mutated to asparagine at 122 position; Kadcyla: Ado-trastuzumab emtansine, anti-HER2 antibody-drug conjugate; Keytruda: Pembrolizumab, anti-PD-1; Kras: Kirsten rat sarcoma virus oncogene; KS: Kaposi's sarcoma; KSHV: Kaposi sarcoma-associated herpesvirus; Kymriah: Tisagenlecleucel, anti-CD19 CAR-T cells; L chain: Immunoglobulin light chain; L265P: Leucine mutated to proline at 265 position; LANA: Virus latency-associated nuclear antigen from KSHV; LMP1: Latent membrane protein 1; LMP2: Latent membrane protein 2; mAb: Monoclonal antibody; MAC: Membrane attack complex; MAGE: Melanoma-associated antigen; MART: Melanoma antigen recognized by T cells; MCC: Merkel cell carcinoma; MCMV: Murine cytomegalovirus; MHC: Major histocompatibility complex; MYD88: Myeloid differentiation primary response 88; Mylotarg: Gemtuzumab ozogamicin, anti-CD33 antibody-drug conjugate; NHL: Non-Hodgkin's lymphoma; NK: Natural killer; NP: Nucleoprotein; NPC: Nasopharyngeal carcinoma; NS3: Virus nonstructural protein 3 from HCV; NS5A: Virus nonstructural protein 5A from HCV; NYESO1: New York esophageal squamous cell carcinoma-1; Opdivo: Nivolumab, anti-PD-1; OVA: Ovalbumin; PD-1: Programmed cell death receptor-1; PEL: Primary effusion lymphoma; PP65: CMV PP65 antigen; PRAME: Preferentially expressed antigen in melanoma; S37F: Serine mutated to phenylalanine at 37 position; scFv: Single-chain fragment variable; SSX: Synovial sarcoma X; SV40: Simian virus 40; TARP: TCR gamma alternate reading frame protein; Tax: The viral transactivator; TCR: T cell receptor; TNFa: Tumor necrosis factor alpha; TP53: Tumor protein p53; vFLIP: Virus FLICE/caspase-8-inhibitory protein from KSHV; VGPCR: Virus active G proteincoupled receptor homolog from KSHV; VH: Heavy chain variable; vIRF-1: Virus IFN-inducible factor from KSHV; VL: Light chain variable; VSV: Vesicular stomatic virus; WT-1: Wilms tumor gene-1; Yervoy: Ipilimumab, anti-CTLA-4

\section{Acknowledgements}

Not applicable

\section{Authors' contributions}

JW and QH designed the study. ZL, ZL, YL, and XZ provided critical suggestions. JW and $\mathrm{QH}$ analyzed the data and wrote the paper. All authors read and approved the final manuscript.

\section{Funding}

This study was conducted with support from the National Natural Science Foundation of China (No. 81570189 and 81670480), Innovation Team Project of Guangzhou Education Bureau (201831828), and Science and Technology Program of Guangzhou (201902020001).

\section{Availability of data and materials}

The dataset supporting the conclusions of this article is included within the article.

\section{Ethics approval and consent to participate}

Not applicable

\section{Consent for publication}

Not applicable

\section{Competing interests}

The authors declare that they have no competing interests.

\section{Author details}

'Department of Center Laboratory, The Fifth Affiliated Hospital of Guangzhou Medical University, Guangzhou 510700, China. ${ }^{2}$ Department of Lymphoma and Myeloma, Division of Cancer Medicine, The University of Texas MD Anderson Cancer Center, 1414 Holcombe Boulevard, Houston, TX 77030, USA.

Received: 6 July 2019 Accepted: 3 September 2019

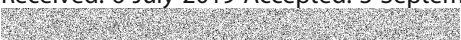

\section{References}

1. Couzin-Frankel J. Breakthrough of the year 2013. Cancer immunotherapy. Science. 2013;342(6165):1432-3.

2. Almagro JC, et al. Progress and challenges in the design and clinical development of antibodies for cancer therapy. Front Immunol. 2017;8:1751.

3. Strohl WR. Current progress in innovative engineered antibodies. Protein Cell. 2018:9(1):86-120.

4. Rexer BN, Arteaga CL. Intrinsic and acquired resistance to HER2-targeted therapies in HER2 gene-amplified breast cancer: mechanisms and clinical implications. Crit Rev Oncog. 2012;17(1):1-16.

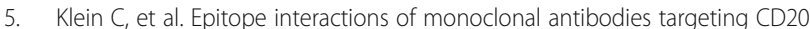
and their relationship to functional properties. MAbs. 2013;5(1):22-33.

6. Beck A, et al. Strategies and challenges for the next generation of antibodydrug conjugates. Nat Rev Drug Discov. 2017;16(5):315-37.

7. Dahan R, Reiter Y. T-cell-receptor-like antibodies - generation, function and applications. Expert Rev Mol Med. 2012;14:e6.

8. Neefjes J, et al. Towards a systems understanding of MHC class I and MHC class II antigen presentation. Nat Rev Immunol. 2011;11:823.

9. Smith-Garvin JE, Koretzky GA, Jordan MS. T cell activation. Annu Rev Immunol. 2009:27(1):591-619.

10. Chang AY, et al. Opportunities and challenges for TCR mimic antibodies in cancer therapy. Expert Opin Biol Ther. 2016;16(8):979-87.

11. Inaguma $Y$, et al. Construction and molecular characterization of a T-cell receptor-like antibody and CAR-T cells specific for minor histocompatibility antigen HA-1H. Gene Ther. 2014;21(6):575-84.

12. Wulfing C, Pluckthun A. Correctly folded T-cell receptor fragments in the periplasm of Escherichia coli. Influence of folding catalysts. J Mol Biol. 1994; 242(5):655-69.

13. Corr M, et al. T cell receptor-MHC class I peptide interactions: affinity, kinetics, and specificity. Science. 1994;265(5174):946-9.

14. Matsui $K$, et al. Kinetics of T-cell receptor binding to peptide/l-Ek complexes: correlation of the dissociation rate with T-cell responsiveness. Proc Natl Acad Sci U S A. 1994;91(26):12862-6.

15. Dao T, et al. Targeting the intracellular WT1 oncogene product with a therapeutic human antibody. Sci Transl Med. 2013;5(176):176ra33.

16. Denkberg $G$, et al. Selective targeting of melanoma and APCs using a recombinant antibody with TCR-like specificity directed toward a melanoma differentiation antigen. J Immunol. 2003;171(5):2197-207.

17. Dao $T$, et al. Therapeutic bispecific T-cell engager antibody targeting the intracellular oncoprotein WT1. Nat Biotechnol. 2015;33(10):1079-86.

18. Trenevska I, Li D, Banham AH. Therapeutic antibodies against intracellular tumor antigens. Front Immunol. 2017;8:1001.

19. Dubrovsky $L$, et al. $T$ cell receptor mimic antibodies for cancer therapy. Oncoimmunology. 2016;5(1):e1049803.

20. Chames $P$, et al. Direct selection of a human antibody fragment directed against the tumor T-cell epitope HLA-A1-MAGE-A1 from a nonimmunized phage-Fab library. Proc Natl Acad Sci. 2000;97(14):7969-74.

21. Willemsen RA, et al. A phage display selected fab fragment with MHC class I-restricted specificity for MAGE-A1 allows for retargeting of primary human T lymphocytes. Gene Ther. 2001;8(21):1601-8.

22. Chames $P$, et al. TCR-like human antibodies expressed on human CTLS mediate antibody affinity-dependent cytolytic activity. J Immunol. 2002; 169(2):1110-8.

23. Denkberg G, Klechevsky E, Reiter Y. Modification of a tumor-derived peptide at an HLA-A2 anchor residue can alter the conformation of the MHCpeptide complex: probing with TCR-like recombinant antibodies. J Immunol. 2002;169(8):4399-407. 
24. Hoogenboom, H., Y. Reiter, MHC-peptide complex binding ligands. 2003, Google Patents.

25. Zhang G, et al. Anti-melanoma activity of $T$ cells redirected with a TCR-like chimeric antigen receptor. Sci Rep. 2014;4:3571.

26. Klechevsky $\mathrm{E}$, et al. Antitumor activity of immunotoxins with T-cell receptorlike specificity against human melanoma xenografts. Cancer Res. 2008; 68(15):6360-7

27. Lev A, et al. Isolation and characterization of human recombinant antibodies endowed with the antigen-specific, major histocompatibility complex-restricted specificity of T cells directed toward the widely expressed tumor T-cell epitopes of the telomerase catalytic subunit. Cancer Res. 2002;62(11):3184-94

28. Cohen CJ, et al. Direct detection and quantitation of a distinct T-cell epitope derived from tumor-specific epithelial cell-associated mucin using human recombinant antibodies endowed with the antigen-specific, major histocompatibility complex-restricted specificity of T cells. Cancer Res. 2002; 62(20):5835-44.

29. Held G, et al. Dissecting cytotoxic T cell responses towards the NY-ESO-1 protein by peptide/MHC-specific antibody fragments. Eur J Immunol. 2004 34(10):2919-29.

30. Bernardeau $\mathrm{K}$, et al. Assessment of CD8 involvement in T cell clone avidity by direct measurement of HLA-A2/Mage3 complex density using a highaffinity TCR like monoclonal antibody. Eur J Immunol. 2005;35(10):2864-75.

31. Wittman VP, et al. Antibody targeting to a class I MHC-peptide epitope promotes tumor cell death. J Immunol. 2006;177(6):4187-95.

32. Neethling FA, et al. Assessing vaccine potency using TCRmimic antibodies. Vaccine. 2008;26(25):3092-102.

33. Weidanz JA, et al. Levels of specific peptide-HLA class I complex predicts tumor cell susceptibility to CTL killing. J Immunol. 2006;177(8):5088-97.

34. Held G, et al. MHC-peptide-specific antibodies reveal inefficient presentation of an HLA-A*0201-restricted, Melan-A-derived peptide after active intracellular processing. Eur J Immunol. 2007:37(7):2008-17.

35. Epel $\mathrm{M}$, et al. Targeting TARP, a novel breast and prostate tumor-associated antigen, with $\mathrm{T}$ cell receptor-like human recombinant antibodies. Eur J Immunol. 2008;38(6):1706-20,

36. Li D, et al. Development of a T-cell receptor mimic antibody against wildtype p53 for cancer immunotherapy. Cancer Res. 2017;77(10):2699-711.

37. Li D, et al. Engineering chimeric human and mouse major histocompatibility complex (MHC) class I tetramers for the production of Tcell receptor (TCR) mimic antibodies. PLoS One. 2017;12(4):e0176642.

38. Michaeli $Y$, et al. Expression hierarchy of $\mathrm{T}$ cell epitopes from melanoma differentiation antigens: unexpected high level presentation of tyrosinaseHLA-A2 Complexes revealed by peptide-specific, MHC-restricted, TCR-like antibodies. J Immunol. 2009;182(10):6328-41.

39. Verma B, et al. Direct discovery and validation of a peptide/MHC epitope expressed in primary human breast cancer cells using a TCRm monoclonal antibody with profound antitumor properties. Cancer Immunol Immunother. 2010;59(4):563-73.

40. Hawkins $\mathrm{O}$, et al. An HLA-presented fragment of macrophage migration inhibitory factor is a therapeutic target for invasive breast cancer. J Immunol. 2011;186(11):6607-16.

41. Sergeeva A, et al. An anti-PR1/HLA-A2 T-cell receptor-like antibody mediates complement-dependent cytotoxicity against acute myeloid leukemia progenitor cells. Blood. 2011;117(16):4262-72.

42. Ma Q, et al. A novel TCR-like CAR with specificity for PR1/HLA-A2 effectively targets myeloid leukemia in vitro when expressed in human adult peripheral blood and cord blood T cells. Cytotherapy. 2016;18(8):985-94.

43. Oren $\mathrm{R}$, et al. Functional comparison of engineered $T$ cells carrying a native TCR versus TCR-like antibody-based chimeric antigen receptors indicates affinity/avidity thresholds. J Immunol. 2014;193(11):5733-43.

44. Zhao $\mathrm{Q}$, et al. Affinity maturation of T-cell receptor-like antibodies for Wilms tumor 1 peptide greatly enhances therapeutic potential. Leukemia. 2015; 29(11):2238-47.

45. Chang AY, et al. A therapeutic T cell receptor mimic antibody targets tumorassociated PRAME peptide/HLA-I antigens. J Clin Invest. 2017;127(7):2705-18.

46. Cohen $\mathrm{CJ}$, et al. Direct phenotypic analysis of human MHC class I antigen presentation: visualization, quantitation, and in situ detection of human viral epitopes using peptide-specific, MHC-restricted human recombinant antibodies. J Immunol. 2003;170(8):4349-61.

47. Yamano $Y$, et al. Increased expression of human T lymphocyte virus type I (HTLV-I) Tax11-19 peptide-human histocompatibility leukocyte antigen
A*201 complexes on CD4+ CD25+ T cells detected by peptide-specific, major histocompatibility complex-restricted antibodies in patients with HTLV-I-associated neurologic disease. J Exp Med. 2004;199(10):1367-77.

48. Biddison WE, et al. Tax and M1 peptide/HLA-A2-specific Fabs and T cell receptors recognize nonidentical structural features on peptide/HLA-A2 complexes. J Immunol. 2003;171(6):3064-74.

49. Sastry KSR, et al. Targeting hepatitis B virus-infected cells with a T-cell receptor-like antibody. J Virol. 2011;85(5):1935-42.

50. Herschhorn A, Marasco WA, Hizi A. Antibodies and lentiviruses that specifically recognize a T cell epitope derived from HIV-1 Nef protein and presented by HLA-C. J Immunol. 2010;185(12):7623-32.

51. Weidanz JA, et al. Development and implementation of a direct detection, quantitation and validation system for class I MHC self-peptide epitopes. J Immunol Methods. 2007;318(1-2):47-58.

52. Nunoya J, et al. Short communication: generation of recombinant monoclonal antibodies against an immunodominant HLA-A*2402-restricted HIV type 1 CTL epitope. AIDS Res Hum Retrovir. 2009;25(9):897-904.

53. Makler $\mathrm{O}$, et al. Direct visualization of the dynamics of antigen presentation in human cells infected with cytomegalovirus revealed by antibodies mimicking TCR specificity. Eur J Immunol. 2010;40(6):1552-65.

54. Tashiro H, Brenner MK. Immunotherapy against cancer-related viruses. Cell Res. 2017;27(1):59-73.

55. Nayersina R, et al. HLA A2 restricted cytotoxic T lymphocyte responses to multiple hepatitis B surface antigen epitopes during hepatitis B virus infection. J Immunol. 1993;150(10):4659-71.

56. Mizukoshi $\mathrm{E}$, et al. Cellular immune responses to the hepatitis B virus polymerase. J Immunol. 2004;173(9):5863-71.

57. Gao J, et al. Construction and characterization of an HCV-derived multiepitope peptide antigen containing B-cell HVR1 mimotopes and T-cell conserved epitopes. Sci China C Life Sci. 2006:49(5):490-9.

58. Ressing ME, et al. Differential binding of viral peptides to HLA-A2 alleles. Implications for human papillomavirus type 16 E7 peptide-based vaccination against cervical carcinoma. Eur J Immunol. 1999;29(4):1292-303.

59. Kozako T, et al. Target epitopes of HTLV-1 recognized by class I MHC-restricted cytotoxic T lymphocytes in patients with myelopathy and spastic paraparesis and infected patients with autoimmune disorders. J Med Virol. 2011;83(3):501-9.

60. Mesri EA, Feitelson MA, Munger K. Human viral oncogenesis: a cancer hallmarks analysis. Cell Host Microbe. 2014;15(3):266-82.

61. Kondo $\mathrm{E}$, et al. Identification of novel CTL epitopes of CMV-pp65 presented by a variety of HLA alleles. Blood. 2004;103(2):630-8.

62. Sahay B, Nguyen CQ, Yamamoto JK. Conserved HIV epitopes for an effective HIV vaccine. J Clin Cell Immunol. 2017:8(4):518.

63. Luo GG, Ou J-hJ. Oncogenic viruses and cancer. Virol Sin. 2015;30(2):83-4.

64. Moore PS, Chang Y. Why do viruses cause cancer? Highlights of the first century of human tumour virology. Nat Rev Cancer. 2010;10:878.

65. Stratton MR, Campbell PJ, Futreal PA. The cancer genome. Nature. 2009; 458(7239):719-24.

66. Futreal PA, et al. A census of human cancer genes. Nat Rev Cancer. 2004; 4(3):177-83.

67. Schumacher TN, Scheper W, Kvistborg P. Cancer neoantigens. Annu Rev Immunol. 2019:37(1):173-200.

68. Robbins PF, et al. A mutated beta-catenin gene encodes a melanomaspecific antigen recognized by tumor infiltrating lymphocytes. J Exp Med. 1996;183(3):1185-92.

69. Echchakir $\mathrm{H}$, et al. A point mutation in the alpha-actinin-4 gene generates an antigenic peptide recognized by autologous cytolytic T lymphocytes on a human lung carcinoma. Cancer Res. 2001;61(10):4078-83.

70. Gaudin C, et al. A hsp70-2 mutation recognized by CTL on a human renal cell carcinoma. J Immunol. 1999;162(3):1730-8.

71. Kubuschok B, et al. Naturally occurring T-cell response against mutated p21 ras oncoprotein in pancreatic cancer. Clin Cancer Res. 2006;12(4):1365-72.

72. Dubois S, et al. Biological and clinical relevance of associated genomic alterations in MYD88 L265P and non-L265P-mutated diffuse large B-cell Iymphoma: analysis of 361 cases. Clin Cancer Res. 2017:23(9):2232-44.

73. Yotnda P, et al. Cytotoxic T cell response against the chimeric p210 BCR$A B L$ protein in patients with chronic myelogenous leukemia. J Clin Invest. 1998;101(10):2290-6

74. Yarchoan $\mathrm{M}$, et al. Targeting neoantigens to augment antitumour immunity. Nat Rev Cancer. 2017;17:209

75. Guo Y, Lei K, Tang L. Neoantigen vaccine delivery for personalized anticancer immunotherapy. Front Immunol. 2018;9:1499. 
76. De Backer O, et al. Characterization of the GAGE genes that are expressed in various human cancers and in normal testis. Cancer Res. 1999;59(13): 3157-65.

77. Chomez $\mathrm{P}$, et al. An overview of the MAGE gene family with the identification of all human members of the family. Cancer Res. 2001;61(14): 5544-51.

78. Ping Y, Liu C, Zhang Y. T-cell receptor-engineered T cells for cancer treatment: current status and future directions. Protein Cell. 2018;9(3):254-66.

79. Thomas R, et al. NY-ESO-1 based immunotherapy of cancer: current perspectives. Front Immunol. 2018;9:947.

80. Bethune MT, et al. Isolation and characterization of NY-ESO-1-specific T cell receptors restricted on various MHC molecules. Proc Natl Acad Sci. 2018; 115(45):E10702-11.

81. Krishnadas DK, Bai F, Lucas KG. Cancer testis antigen and immunotherapy. ImmunoTargets Ther. 2013;2:11-9.

82. Scanlan MJ, et al. Cancer/testis antigens: an expanding family of targets fo cancer immunotherapy. Immunol Rev. 2002;188(1):22-32.

83. Brichard $V$, et al. The tyrosinase gene codes for an antigen recognized by autologous cytolytic T lymphocytes on HLA-A2 melanomas. J Exp Med. 1993;178(2):489-95.

84. Duffy MJ. Carcinoembryonic antigen as a marker for colorectal cancer: is it clinically useful? Clin Chem. 2001;47(4):624-30.

85. Coulie $P G$, et al. Tumour antigens recognized by $T$ lymphocytes: at the core of cancer immunotherapy. Nat Rev Cancer. 2014;14:135.

86. Parkhurst MR, et al. T cells targeting carcinoembryonic antigen can mediate regression of metastatic colorectal cancer but induce severe transient colitis. Mol Ther. 2011;19(3):620-6.

87. Johnson LA, et al. Gene therapy with human and mouse T-cell receptors mediates cancer regression and targets normal tissues expressing cognate antigen. Blood. 2009;114(3):535-46.

88. Rafei H, Kantarjian HM, Jabbour EJ. Recent advances in the treatment of acute lymphoblastic leukemia. Leuk Lymphoma. 2019; p.1-16.

89. Brudno JN, Kochenderfer JN. Chimeric antigen receptor T-cell therapies for lymphoma. Nat Rev Clin Oncol. 2018;15(1):31-46.

90. Neelapu SS. Managing the toxicities of CAR T-cell therapy. Hematol Oncol. 2019:37(Suppl 1):48-52.

91. Novellino L, Castelli C, Parmiani G. A listing of human tumor antigens recognized by T cells: March 2004 update. Cancer Immunol Immunother. 2005;54(3):187-207.

92. Blum JS, Wearsch PA, Cresswell P. Pathways of antigen processing. Annu Rev Immunol. 2013;31:443-73.

93. Corse E, Gottschalk RA, Allison JP. Strength of TCR-peptide/MHC interactions and in vivo T cell responses. J Immunol. 2011;186(9):5039-45.

94. Kunert A, et al. TCR-engineered T cells meet new challenges to treat solid tumors: choice of antigen, $T$ cell fitness, and sensitization of tumor milieu. Front Immunol. 2013;4:363.

95. Wylie DE, Klinman NR. The murine B cell repertoire responsive to an influenza-infected syngeneic cell line. J Immunol. 1981;127(1):194-8.

96. Wylie DE, Sherman LA, Klinman NR. Participation of the major histocompatibility complex in antibody recognition of viral antigens expressed on infected cells. J Exp Med. 1982;155(2):403-14

97. Froscher BG, Klinman NR. Immunization with SV40-transformed cells yields mainly MHC-restricted monoclonal antibodies. J Exp Med. 1986;164(1):196-210.

98. Catipović B, et al. Major histocompatibility complex conformational epitopes are peptide specific. J Exp Med. 1992;176(6):1611-8.

99. Hogquist KA, Grandea AG, Bevan MJ. Peptide variants reveal how antibodies recognize major histocompatibility complex class I. Eur J Immunol. 1993; 23(11):3028-36.

100. Syed ON, et al. Cancer-testis and melanocyte-differentiation antigen expression in malignant glioma and meningioma. J Clin Neurosci. 2012; 19(7):1016-21.

101. Pastan I, Kreitman RJ. Immunotoxins in cancer therapy. Curr Opin Investig Drugs. 2002;3(7):1089-91.

102. Verma B, et al. TCR mimic monoclonal antibodies induce apoptosis of tumor cells via immune effector-independent mechanisms. J Immunol. 2011;186(5):3265-76

103. Zheng P-P, Kros JM, Li J. Approved CAR T cell therapies: ice bucket challenges on glaring safety risks and long-term impacts. Drug Discov Today. 2018;23(6):1175-82.

104. Dubrow $R$, et al. HIV infection, aging, and immune function: implications for cancer risk and prevention. Curr Opin Oncol. 2012;24(5):506-16.
105. McMichael AJ, et al. The immune response during acute HIV-1 infection: clues for vaccine development. Nat Rev Immunol. 2010;10(1):11-23.

106. Aharoni $\mathrm{R}$, et al. Immunomodulation of experimental allergic encephalomyelitis by antibodies to the antigen-la complex. Nature. 1991; 351:147.

107. Litvak-Greenfeld D, Benhar I. Risks and untoward toxicities of antibodybased immunoconjugates. Adv Drug Deliv Rev. 2012;64(15):1782-99.

108. Kreitman RJ. Recombinant immunotoxins containing truncated bacterial toxins for the treatment of hematologic malignancies. BioDrugs. 2009; 23(1):1-13.

109. Stewart-Jones $\mathrm{G}$, et al. Rational development of high-affinity T-cell receptorlike antibodies. Proc Natl Acad Sci U S A. 2009;106(14):5784-8.

110. Fernandez-Miguel $\mathrm{G}$, et al. Multivalent structure of an alphabetaT cell receptor. Proc Natl Acad Sci U S A. 1999;96(4):1547-52.

111. Kuball J, et al. Facilitating matched pairing and expression of TCR chains introduced into human T cells. Blood. 2007;109(6):2331-8.

112. Zhao J, et al. Universal CARs, universal T cells, and universal CAR T cells. J Hematol Oncol. 2018;11(1):132.

113. Maude SL, et al. Chimeric antigen receptor T cells for sustained remissions in leukemia. N Engl J Med. 2014;371(16):1507-17.

114. Maude SL, et al. Tisagenlecleucel in children and young adults with B-cell lymphoblastic leukemia. N Engl J Med. 2018;378(5):439-48.

115. Maude SL, et al. CD19-targeted chimeric antigen receptor T-cell therapy for acute lymphoblastic leukemia. Blood. 2015;125(26):4017-23.

116. Li J, et al. Chimeric antigen receptor T cell (CAR-T) immunotherapy for solid tumors: lessons learned and strategies for moving forward. J Hematol Oncol. 2018;11(1):22

117. Pardoll DM. The blockade of immune checkpoints in cancer immunotherapy. Nat Rev Cancer. 2012;12:252.

118. Havel JJ, Chowell D, Chan TA. The evolving landscape of biomarkers for checkpoint inhibitor immunotherapy. Nat Rev Cancer. 2019;19(3):133-50.

119. Wolchok JD, et al. Nivolumab plus ipilimumab in advanced melanoma. $N$ Engl J Med. 2013;369(2):122-33.

120. Robert C, et al. Pembrolizumab versus ipilimumab in advanced melanoma. N Engl J Med. 2015;372(26):2521-32.

121. Rosenberg SA, Yang JC, Restifo NP. Cancer immunotherapy: moving beyond current vaccines. Nat Med. 2004;10(9):909-15.

122. Banchereau J, Palucka K. Cancer vaccines on the move. Nat Rev Clin Oncol. 2017;15:9.

123. Marin-Acevedo JA, et al. Cancer immunotherapy beyond immune checkpoint inhibitors. J Hematol Oncol. 2018;11(1):8.

124. Petrulio CA, Kim-Schulze S, Kaufman HL. The tumour microenvironment and implications for cancer immunotherapy. Expert Opin Biol Ther. 2006;6(7): 671-84

125. Leone $\mathrm{P}$, et al. MHC class I antigen processing and presenting machinery: organization, function, and defects in tumor cells. JNCl. 2013;105(16):1172-87.

126. Garrido F, et al. The urgent need to recover MHC class I in cancers for effective immunotherapy. Curr Opin Immunol. 2016:39:44-51.

127. Garrido F, Cabrera T, Aptsiauri N. "Hard" and "soft" lesions underlying the HLA class I alterations in cancer cells: implications for immunotherapy. Int J Cancer. 2010;127(2):249-56.

128. Binnewies $M$, et al. Understanding the tumor immune microenvironment (TIME) for effective therapy. Nat Med. 2018;24(5):541-50.

129. Liu Y, Cao X. Immunosuppressive cells in tumor immune escape and metastasis. J Mol Med (Berl). 2016;94(5):509-22.

130. Kitamura T, Qian BZ, Pollard JW. Immune cell promotion of metastasis. Nat Rev Immunol. 2015:15(2):73-86.

\section{Publisher's Note}

Springer Nature remains neutral with regard to jurisdictional claims in published maps and institutional affiliations. 